\title{
Aerial Co-robots of Future: How Far We Are
}

\author{
Naira Hovakimyan \\ Department of Mechanical Science and Engineering \\ University of Illinois at Urbana-Champaign \\ USA
}

The presentation will give a historical overview of flight control technology from its inception till its maturation. Parallel developments in aerial robotics will be reviewed from the perspective of aerospace industry standards, prioritizing safety, resilience and reliability of operations. Special focus will be placed on cooperative control of UAVs for various mission scenarios in military operations. Flight tests of a subscale commercial jet at NASA and Learjet at Edwards Air Force base will be used to demonstrate the efficiency of the methods developed over the past ten years. Lessons learned will be summarized, and the opportunities in public safety, elderly care, package delivery, precision farming and digital agriculture will be discussed. 